\title{
Nonlinear filtering in object and Fourier space in a joint transform optical correlator: comparison and experimental realization
}

\author{
Santiago Vallmitjana, Arturo Carnicer, Estela Martín-Badosa, and Ignacio Juvells
}

\begin{abstract}
The use of different kinds of nonlinear filtering in a joint transform correlator are studied and compared. The study is divided into two parts, one corresponding to object space and the second to the Fourier domain of the joint power spectrum. In the first part, phase and inverse filters are computed; their inverse Fourier transforms are also computed, thereby becoming the reference in the object space. In the Fourier space, the binarization of the power spectrum is realized and compared with a new procedure for removing the spatial envelope. All cases are simulated and experimentally implemented by a compact joint transform correlator.

Key words: Joint transform correlator, pattern recognition, nonlinear filtering.
\end{abstract}

\section{Introduction}

Since the development of the VanderLugt classical matched filter, ${ }^{1}$ several correlation filters for pattern recognition have been proposed to improve recognition capability. Most of these filters are based mainly on modifications of the amplitude or phase of the original matched filter, and in this sense they perform as nonlinear filters. Although phase-only filters ${ }^{2}$ (POF's) and inverse filters ${ }^{3}$ (IF's) usually operate in a VanderLugt architecture, it is possible by computing and codification methods to use them as a reference in a joint transform correlator (JTC). In this context we can designate them as object-space nonlinear filters.

The use of nonlinearities in the joint power spectrum (JPS) in a JTC has also been proved to be a good method for improving discrimination capability. ${ }^{4,5}$ Although in multiobject scenes the binarization of the JPS with a fixed threshold could induce the presence of peaks that produce false alarms, ${ }^{6,7}$ recently this problem has been solved by the use of more sophisticated methods. ${ }^{8}$

The aim of this work is the comparison of different nonlinear filtering methods in object and Fourier

The authors are with the Laboratori d'Optica, Departament de Física Aplicada i Electrònica, Universitat de Barcelona, Diagonal 647, Barcelona E-08028, Spain.

Received 7 December 1994; revised manuscript received 7 December 1994.

0003-6935/95/203942-08\$06.00/0.

(c) 1995 Optical Society of America. space. The results are compared in all cases described, through digital simulation and optical experimental realizations, which have been carried out with a JTC implemented with an electrically addressed spatial light modulator (SLM) in the input plane.

\section{Nonlinearities in Object Space}

POF's and IF's can be considered as nonlinear transformations of a matched filter. Usually these filters are used in the frequency domain in a VanderLugt architecture, although they can be employed in the object space, thereby becoming the reference in a JTC setup. To obtain the filters in object space, the inverse Fourier transform of the filter defined in the frequency domain has to be calculated. These filters in object space are real functions that take positive and negative values in different zones. This implies that a codification is necessary because of the negative values. Another problem involved in the implementation is filter normalization, because the filters usually have a lower transmission than the scene does.

\section{A. Phase-Only and Inverse Filters in a Joint Transform Correlator}

The POF, suggested by Horner and Gianino, ${ }^{2}$ is a method for improving the correlation based on the fact that the phase of a Fourier transform contains most of the significant information of the input. The main advantage is that it has much sharper correlation peaks than the matched filter does. 
The POF is defined as

$$
H_{\mathrm{POF}}(u, v)=\exp \left[-i \phi_{R}(u, v)\right],
$$

where $H_{R}(u, v)=\left|H_{R}(u, v)\right| \exp \left[i \phi_{R}(u, v)\right]$ is the Fourier transform of the target $h_{R}(x, y)$ or reference.

Another type of spatial filter that will give a deltafunction detection peak in the correlation plane is the $\mathrm{IF}^{3}$ which is defined as

$$
\begin{aligned}
H_{\mathrm{IF}}(u, v) & =\left|H_{R}(u, v)\right|^{-1} \exp \left[-i \phi_{R}(u, v)\right] \\
& =H_{R}{ }^{*}(u, v)\left|H_{R}(u, v)\right|^{-2} .
\end{aligned}
$$

However, the IF has severe limitations associated with mathematical poles and small optical efficiency. In order to avoid the poles, in the points where the modulus is tending to 0 , we have left a constant value, which becomes a phase-only value at these points, ${ }^{9}$ i.e.,

$$
\begin{array}{cl}
\left|H_{R}(u, v)\right|^{-1} \exp [-i \phi(u, v)] & \text { if }\left|H_{R}(u, v)\right|>\epsilon_{T} \\
\epsilon_{T}^{-1} \exp [-i \phi(u, v)] & \text { if }\left|H_{R}(u, v)\right|<\epsilon_{T}
\end{array},
$$

where $\epsilon_{T}$ is an arbitrary threshold to be determined.

\section{B. Filter Normalization: Dynamic Range of the Filters}

In the experimental realization, filter normalization is one of the problems involved in the implementation because the filters usually have a lower transmission than that of the scene. The capacity for detection could be affected, depending on the relative energy of the reference and they scene. They have to be normalized before being displayed with the scene in the modulator of the JTC.

To clarify the question, it is interesting to look at the power spectrum in the Fourier plane. Let $h_{R}(x, y)$ be the function representing the target and $h(x, y)$ be the scene located at $\left(x_{0}, y_{0}\right)$. Let $\left|H_{R}(u, v)\right|$ $\exp \left[i \phi_{R}(u, v)\right]$ and $|H(u, v)| \exp \left[i \phi_{s}(u, v)\right]$ be their Fourier transforms, respectively.

The JPS is described by

$$
\begin{aligned}
I(u, v)= & k^{2}\left|H_{R}(u, v)\right|^{2}+|H(u, v)|^{2} \\
& +2 k\left|H_{R}(u, v)\right||H(u, v)| \\
& \times \cos \left[x_{0} u+y_{0} v+\phi_{s}(u, v)-\phi_{R}(u, v)\right],
\end{aligned}
$$

where $k$ is the multiplicative constant for scaling the filter.

Let us analyze the behavior for extreme values of $k$ : for very small values of $k$ the dynamic range of the filter is insufficient, i.e., $|H(u, v)| \gg k\left|H_{R}(u, v)\right|$, and then $I(u, v) \simeq|H(u, v)|^{2}$. For large values of $k$ the dynamic range will be too considerable and will be $k\left|H_{R}(u, v)\right| \gg|H(u, v)|$ and therefore $I(u, v) \simeq$ $k^{2}\left|H_{R}(u, v)\right|^{2}$. In both cases the term corresponding to the interferences is not noticeable enough, and consequently there are no terms of cross correlation.
The correlation plane is written as

$$
\begin{aligned}
c(x, y)= & k^{2} h_{R}(x, y) \otimes h_{R}(x, y)+h(x, y) \otimes h(x, y) \\
& +\left[k h_{R}(x, y) \otimes h(x, y)\right] \delta\left(x-x_{0}, y-y_{0}\right) \\
& +\left[k h(x, y) \otimes h_{R}(x, y)\right] \delta\left(x+x_{0}, y+y_{0}\right)
\end{aligned}
$$

where $\otimes$ and stand for correlation and convolution product, respectively. From the equations displayed, the importance of the selection of a suitable value of $k$ in the JTC is clear.

To establish a criterion to choose a value of $k$, we have taken into account a parameter of efficiency of the JTC. The parameter $E$ is defined as the quotient between the value of the detection peak and the value at the origin of the peak of the autocorrelation in the output plane.

We use its inverse $\left(E^{-1}\right)$ and thus we want $E^{-1}$ to take minimum values. By superimposing this condition in our problem, we have

$$
\begin{aligned}
E^{-1} & =\frac{\left|c_{0}\right|^{2}}{\left|c_{d}\right|^{2}} \\
& =\frac{\left|k^{2} h_{R}(x, y) \otimes h_{R}(x, y)+h(x, y) \otimes h(x, y)\right|_{(0,0)}{ }^{2}}{\left|k h(x, y) \otimes h_{R}(x, y)\right|_{(0,0)}{ }^{2}}
\end{aligned}
$$

The optimal situation is when $E^{-1}$ is minimum, i.e.,

$$
\frac{\partial E^{-1}}{\partial k}=0
$$

By resolving Eq. (7) we have the value

$$
k^{2}=h(x, y) \otimes h(x, y)_{(0,0)} / h_{R}(x, y) \otimes h_{R}(x, y)_{(0,0)} .
$$

Moreover, the value of the autocorrelation at the origin corresponds to the mean value of the square power of the function; then

$$
\begin{gathered}
h_{R}(x, y) \otimes h_{R}(x, y)_{(0,0)}=\left\langle\left|h_{R}(x, y)\right|^{2}\right\rangle, \\
h(x, y) \otimes h(x, y)_{(0,0)}=\left\langle|h(x, y)|^{2}\right\rangle .
\end{gathered}
$$

Therefore the condition to be imposed on $k$ is

$$
k_{E \min }=\left[\left\langle|h(x, y)|^{2}\right\rangle /\left\langle\left|h_{R}(x, y)\right|^{2}\right\rangle\right]^{1 / 2} .
$$

\section{Filter Codification}

The technique used for codification of negative values of the filters is derived from the method of subtraction based on the decomposition of the function in two parts, the positive and the negative, and encoded in Ronchi gratings with the same frequency but in opposition to phase. This provides the subtraction of amplitudes in Fourier space. 
A real two-dimensional function $h(x, y)$ may be expressed as

$$
h(x, y)=h^{+}(x, y)-h^{-}(x, y),
$$

where

$$
\begin{aligned}
h^{+}(x, y) & =h(x, y) & & \text { when } h(x, y)>0 \\
& =0 & & \text { when } h(x, y)<0 \\
h^{-}(x, y) & =-h(x, y) & & \text { when } h(x, y)<0 \\
& =0 & & \text { when } h(x, y)>0
\end{aligned} .
$$

Both $h^{+}$and $h^{-}$are positive functions and are called bipolar filters.

The transmission of a Ronchi grating may be presented by its Fourier series expansion,

$$
r^{+}(x, y)=1+\sum r_{n} \sin (2 \pi n x / p),
$$

where $p$ is the spatial period. If the Ronchi grating is displaced by $p / 2$, its transmission is

$$
r^{-}(x, y)=1-\sum r_{n} \sin (2 \pi n x / p) .
$$

Note that $r^{+}$and $r^{-}$alternate the values 0 and 1 , respectively, along the $\mathrm{x}$ axis and that they are complementary gratings.

Let us consider the following function:

$$
\begin{aligned}
h_{c}(x, y)= & h^{+}(x, y) r^{+}(x, y)+h^{-}(x, y) r^{-}(x, y) \\
= & {\left[h^{+}(x, y)+h^{-}(x, y)\right]+\left[h^{+}(x, y)-h^{-}(x, y)\right] } \\
& \times \sum r_{n} \sin (2 \pi n x / p) .
\end{aligned}
$$

Its two-dimensional Fourier transform is given by

$$
\begin{aligned}
H_{c}(u, v)= & {\left[H^{+}(u, v)+H^{-}(u, v)\right]+\left[H^{+}(u, v)\right.} \\
& \left.-H^{-}(u, v)\right] \otimes \sum r_{n} \delta(u-n / p) \\
= & {\left[H^{+}(u, v)+H^{-}(u, v)\right]+H(u, v) } \\
& \otimes \sum r_{n} \delta(u-n / p) .
\end{aligned}
$$

Finally, by taking the Fourier transform of only one diffraction order [Eq. (14)], we obtain the desired function $h(x, y)$.

We carry out codification of a digital function by representing each sampled value by using four pixels. Both left pixels are switched on together when the original value is positive. Analogously, the righthand ones are switched on when the value is negative. The other two pixels remain $0 .{ }^{10}$

This codification procedure is a further simplification of the Lee's method of generating holograms by computer. ${ }^{11}$ Lee decomposes a complex-value function into four real and positive components. Burckhardt ${ }^{12}$ has already proposed a simplification in which three components are used. As our filter is a real function, we need to use only two real positive

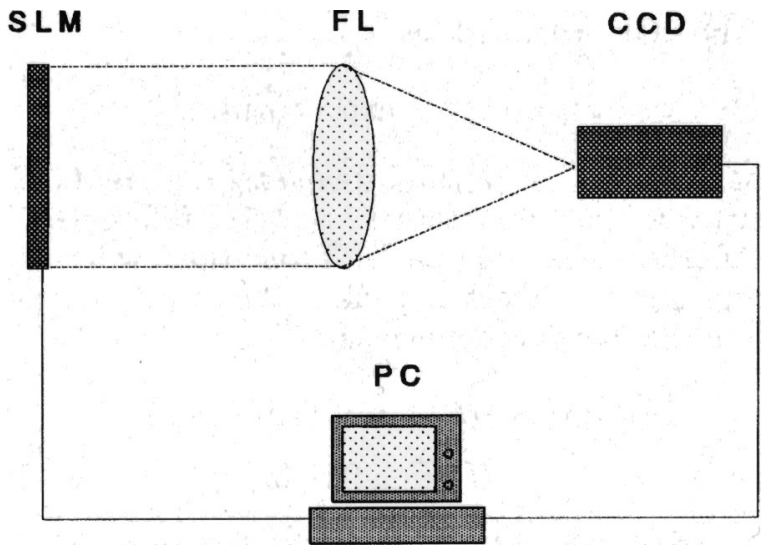

Fig. 1. Single-modulator JTC (FL, Fourier lens).

components, and this simplification reduces the display resolution requirements.

\section{Results}

A real-time JTC that operates with a single liquidcrystal television (LCTV) was implemented as sketched in Fig. 1. ${ }^{13}$ Scene and reference are jointly displayed on the LCTV. The CCD videocamera was connected to an 8-bit digitizer board, and the light distribution was registered in the Fourier plane of the lens system. The LCTV used was obtained from an Epson 1000PS videoprojector. ${ }^{14}$

Once calculated and encoded, the POF is displayed in the liquid-crystal device as a reference, side by side with the scene. A lens system produces a physical Fourier transform in its focal plane, the intensity of which is registered by a CCD videocamera. The JPS is again introduced in the liquid crystal, and, after a second Fourier transform, the CCD detects the correlation.

Figure 2 shows the noncodified original scene. The reference is the lower satellite. Figures 3 and 4 display the appearance of the codified POF and the IF, respectively, both with $128 \times 128$ pixels. In Fig. 5 (a) the digital simulation of correlation with the

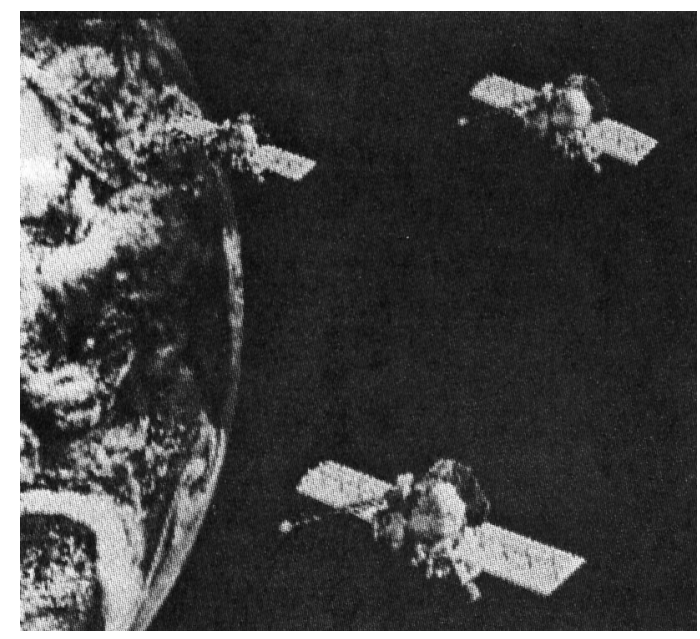

Fig. 2. Scene. The reference is the lower satellite. 


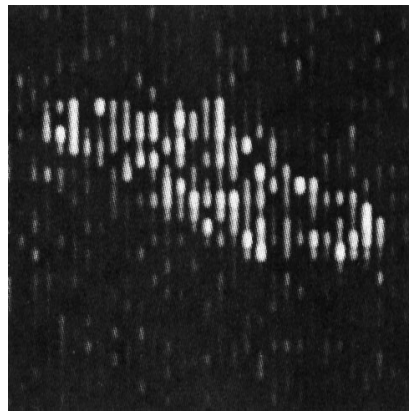

Fig. 3. Codified POF of the reference in object space.

POF is presented to be compared with the results obtained experimentally with the encoded POF in Fig. 5(b). The simulated and the experimental results of the correlations with IF are shown respectively in Figs. 6(a) and 6(b).

\section{Nonlinearities in Fourier Space}

In the past few years, most of the papers published have analyzed different systems in order to increase

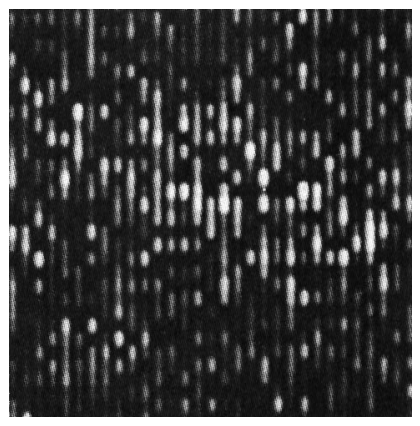

Fig. 4. Codified IF of the reference in object space.

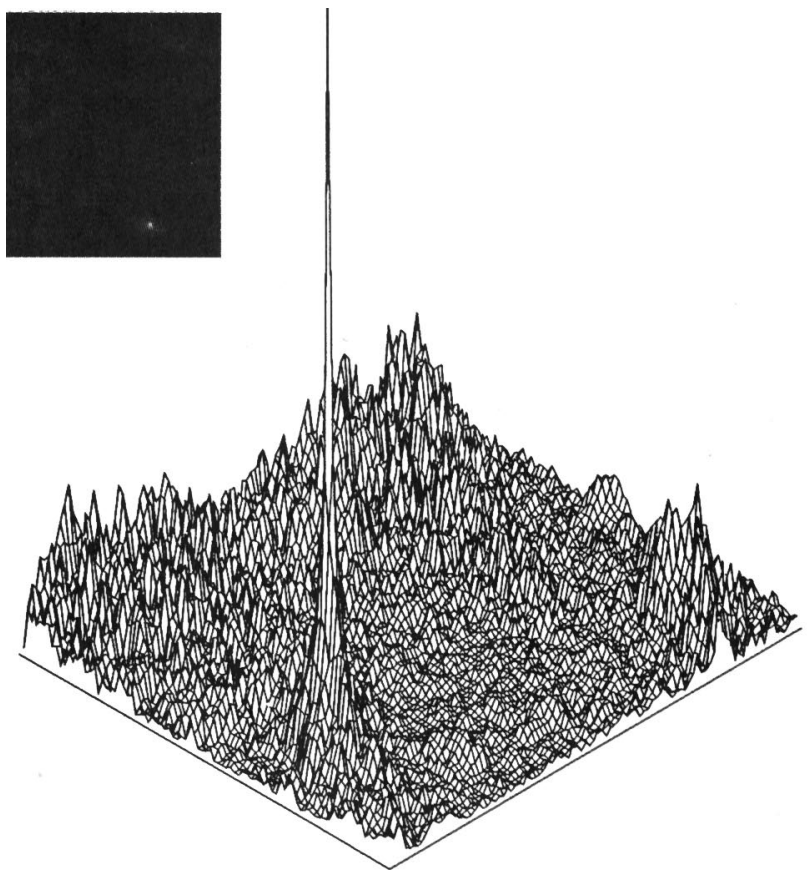

(a)

Fig. 5. Correlation with POF in object space: discrimination capability of the JTC. In particular, the binarization of the JPS has been widely used and has also been shown to be a reliable method. ${ }^{4}$ Several authors have studied various alternatives for defining suitable threshold functions in order to obtain the bipolar power spectrum. Some of these are based on the statistical properties of the JPS or on the elimination of the intraclass terms, which requires a nonnegligible processing time. ${ }^{8}$ The removal of central-correlation terms in real time has been recently proposed. ${ }^{15}$

\section{A. Binarization of the Joint Power Spectrum with} a Variable Threshold

The binary JPS $I_{b}(u, v)$ is obtained when the values +1 or -1 are assigned to the power spectrum $I(u, v)$ :

$$
\begin{aligned}
I(u, v)= & \left|H_{R}(u, v)\right|^{2}+|H(u, v)|^{2}+2\left|H_{R}(u, v)\right||H(u, v)| \\
& \times \cos \left[x_{0} u+y_{0} v+\phi_{s}(u, v)-\phi_{R}(u, v)\right],
\end{aligned}
$$

where $H_{R}(u, v)$ is the Fourier transform of the target. The binarized JPS is defined by

$$
\begin{array}{ll}
I_{b}(u, v)=1 & \text { if } I(u, v)>I_{T}(u, v), \\
I_{b}(u, v)=-1 & \text { if } I(u, v)<I_{T}(u, v),
\end{array}
$$

where $I_{T}(u, v)$ is the variable threshold function defined as

$$
I_{T}(u, v)=\left|H_{R}(u, v)\right|^{2}+|H(u, v)|^{2} .
$$

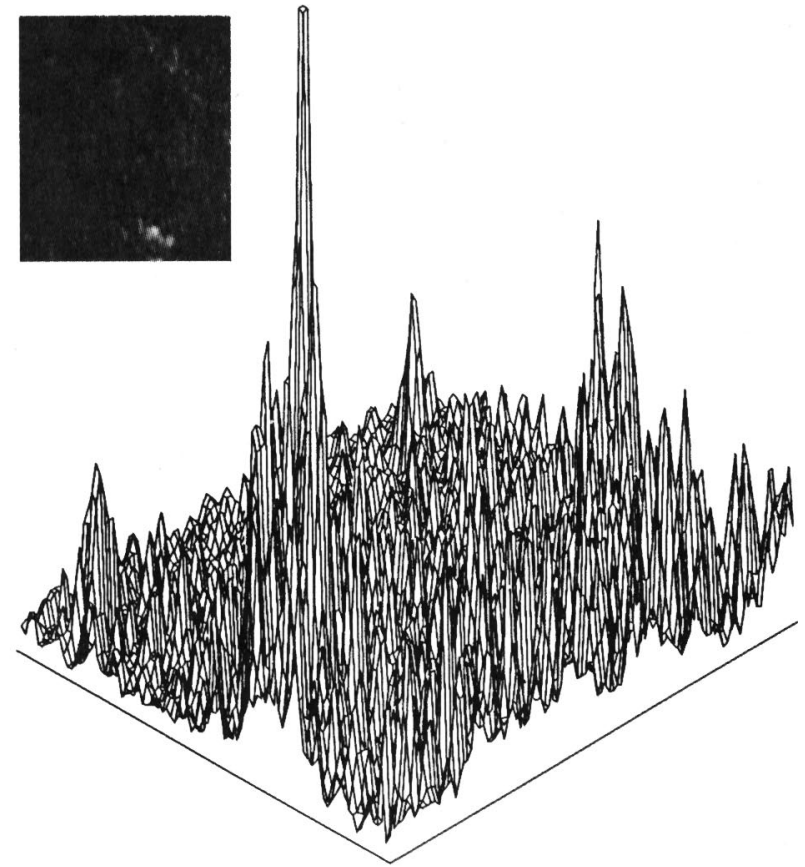

(b)

(a) digital simulation, (b) experimental result. 


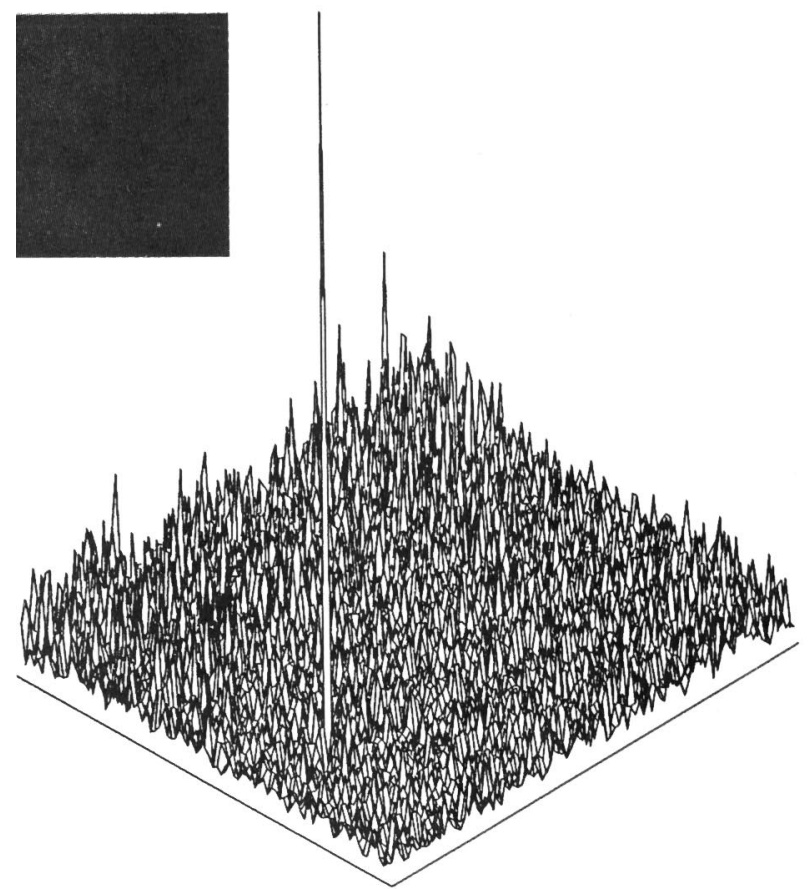

(a)

Fig. 6. Correlation with IF in object space:

The bipolar function $I_{b}(u, v)$ can be expressed as a Fourier expansion ${ }^{4}$ :

$$
\begin{aligned}
I_{b}(u, v)= & \sum_{v} A_{v}\left[u, v ; I_{T}(u, v)\right] \cos \left\{v \left[x_{0} u+y_{0} v\right.\right. \\
& \left.\left.+\phi_{s}(u, v)-\phi_{R}(u, v)\right]\right],
\end{aligned}
$$

where, in this case, the coefficients of the series are constant values, i.e., $A_{v}\left[u, v ; I_{T}(u, v)\right]=A_{v}$, and, consequently, the correlation performs $\delta$ functions. The first-order term produces the correlation based on only the phases of the reference and scene. The other terms could induce redundant self-correlation peaks in the output plane.

\section{B. Spatial Envelope Removal}

We show a simple procedure for eliminating the product $\left|H_{R}(u, v)\right||H(u, v)|$ from the cosine term in Eq. (15). As a consequence, the redundant self-correlation peaks are not obtained. ${ }^{16}$ The main advantage of the experimental implementation lies on the low number of digital operations involved and in the fact that no spatial filters are used. However, its optical implementation requires a gray-scale SLM. The method is based on the direct acquisition of the cosine term in Eq. (15):

$$
\cos \left[x_{0} u+y_{0} v+\phi_{s}(u, v)-\phi_{R}(u, v)\right],
$$

which performs $\delta$-like correlations in $\left(x_{0}, y_{0}\right)$ and $\left(-x_{0},-y_{0}\right)$ if the object is detected. It is important to note that no prior evaluation of $\left|H_{R}(u, v)\right|$ or $|H(u, v)|$ is necessary. We assume that these functions are more slowly variant than the cosines and, conse-

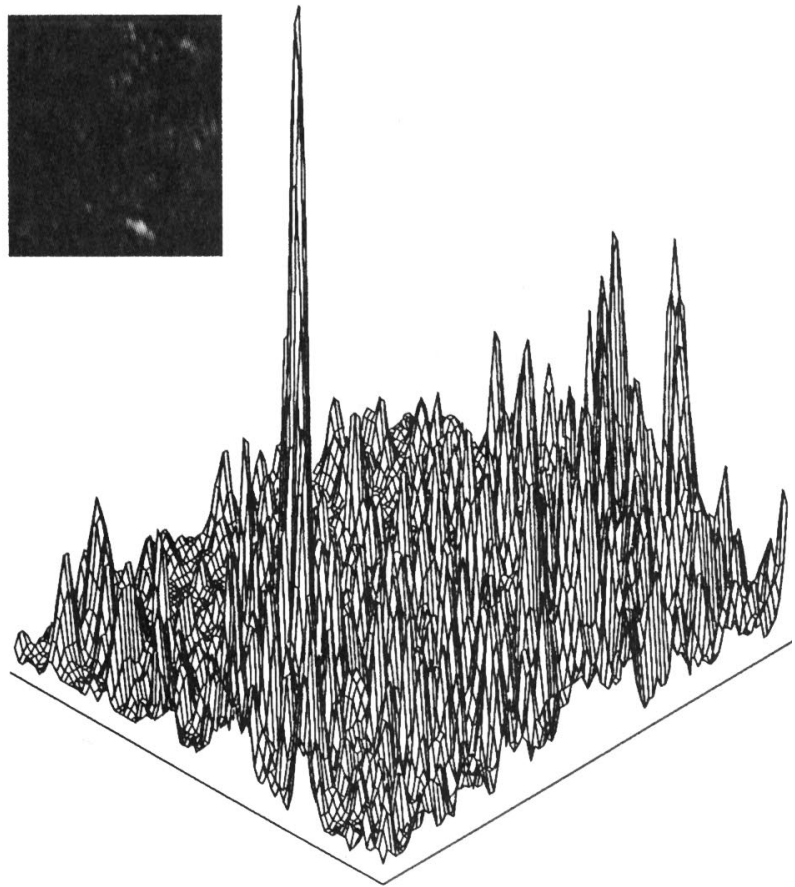

(b)

(a) digital simulation, (b) experimental result.

quently, they can be considered approximately constant in a semiperiod of the cosines in expression (19). Then, by applying a local look-up table to each period of the cosine that scales the values between -1 and +1 , the process is accomplished as shown in Fig. 7 . To apply this procedure, the terms $\left|H_{R}(u, v)\right|^{2}$ and $|H(u, v)|^{2}$ of Eq. (15) should be previously removed, and consequently these distributions have to be regis-

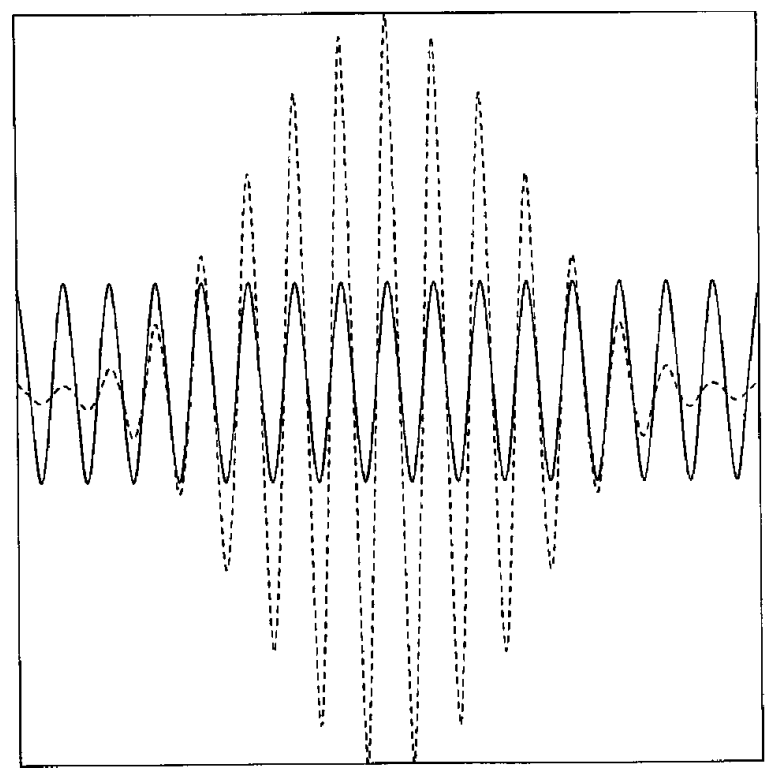

Fig. 7. Schematic procedure of the spatial envelope removal. The dashed curve represents the original sine-modulated function and the solid curve is the result after the application of each local look-up table. 


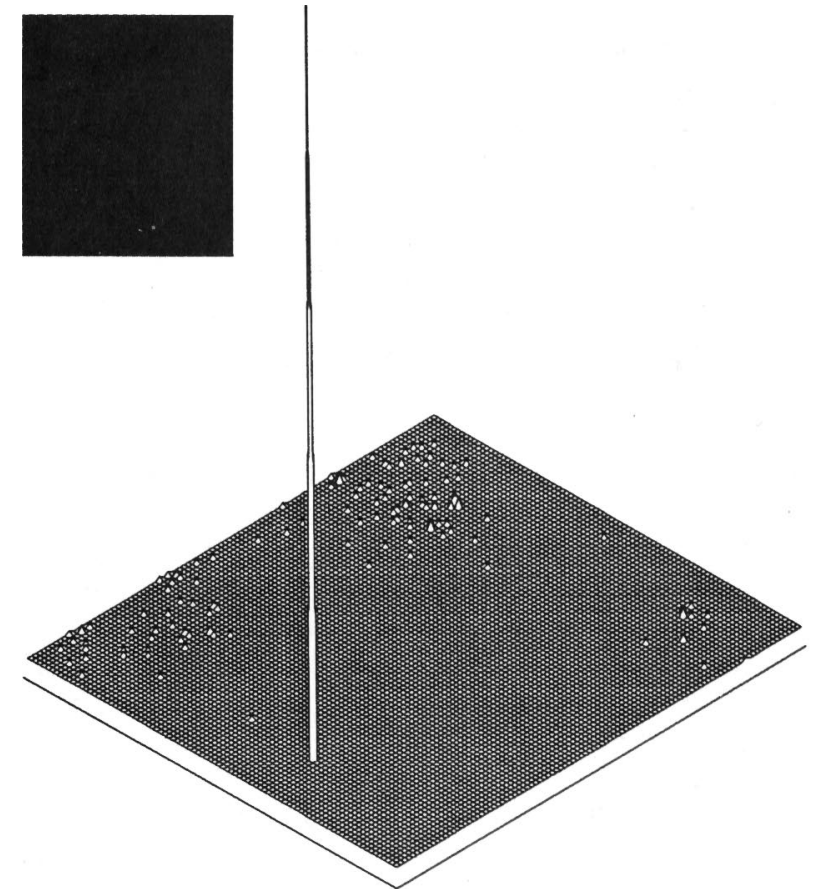

(a)

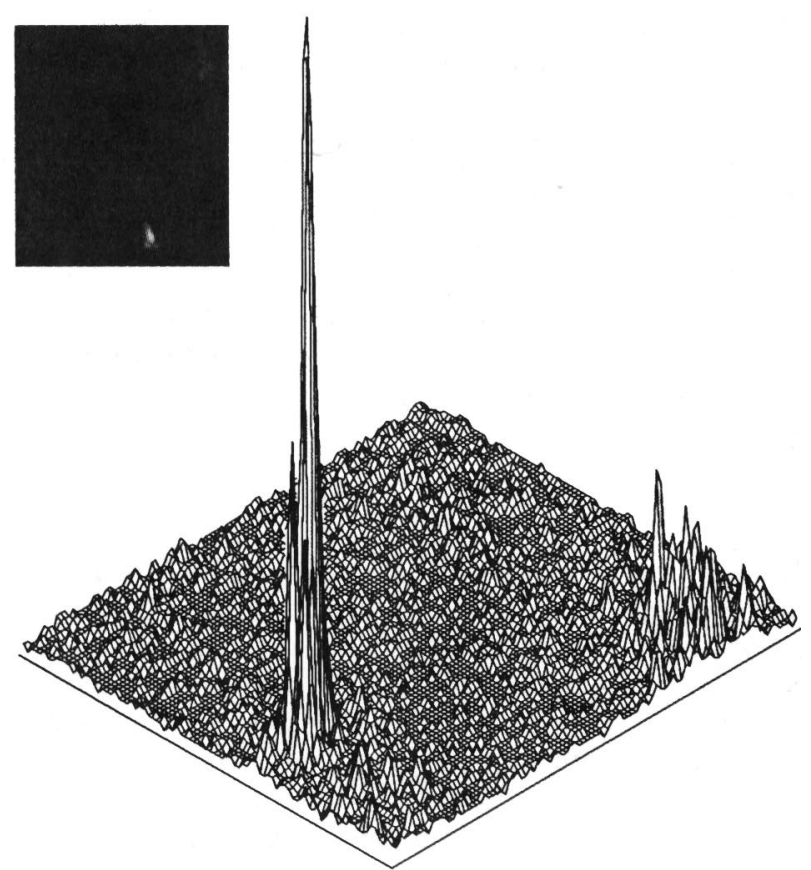

(b)

Fig. 8. Correlation processed with the power spectrum binarized by the threshold function: (a) digital simulation, (b) experimental result.

tered and stored in a computer memory during a previous stage.

\section{Results}

Experimental correlations in which the two methods described above are used have been carried out with the same JTC explained in the first paragraph of
Subsection 2.D. Figure 8(a) corresponds to the simulated correlation when the JPS has been binarized with a variable threshold. Figure $8(b)$ shows the same case experimentally realized. Figure 9(a) corresponds to the simulated correlation when the JPS has been processed by the envelope-free method. Its corresponding experimental case is shown in Fig. 9(b).

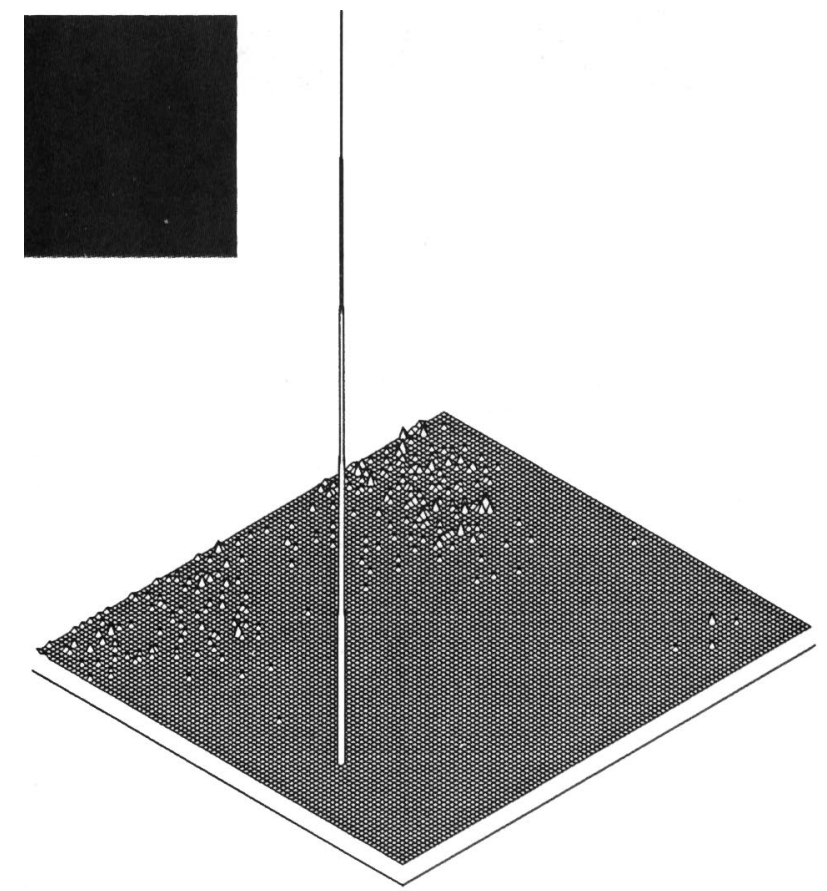

(a)

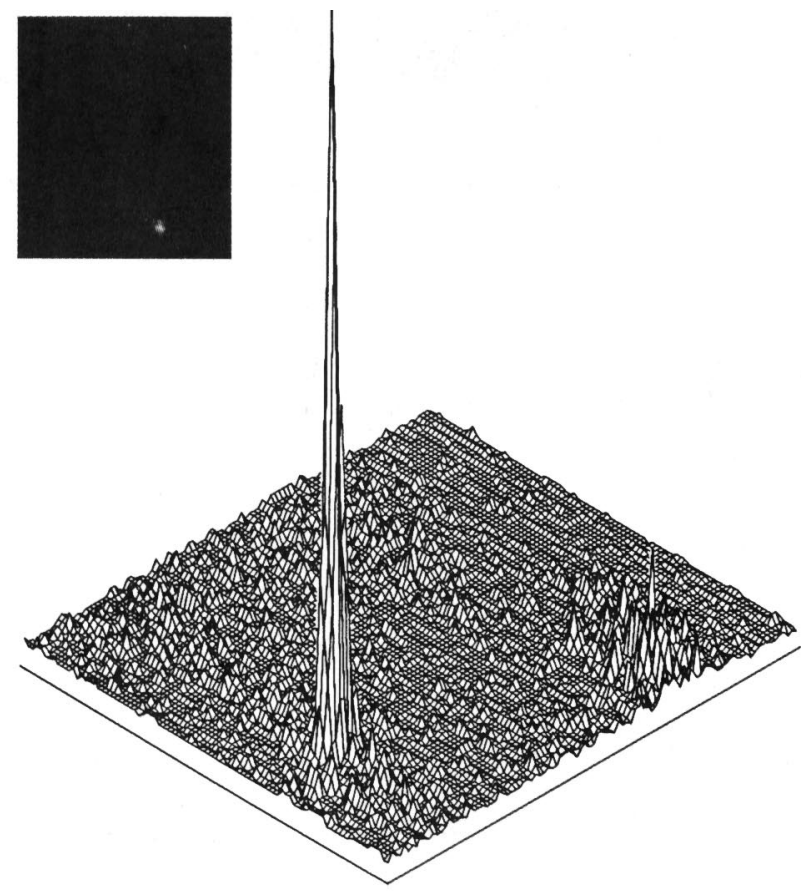

(b)

Fig. 9. Correlation processed with the spatial envelope removal method: (a) digital simulation, (b) experimental result. 
Table 1. Comparison of Different Nonlinear Filtering Methods

\begin{tabular}{lcccc}
\hline \multicolumn{1}{c}{ Parameters } & POF & IF & BJTC $^{a}$ & SER-JTC $^{b}$ \\
\hline Discrimination & 63.5 & 48.5 & 74.4 & 84.3 \\
Peak-to-correlation energy & 0.005 & 0.007 & 0.014 & 0.032 \\
\hline
\end{tabular}

${ }^{a}$ Binary joint transform correlation.

${ }^{b}$ Spatial envelope removal joint transform correlation.

To quantify and summarize the results obtained in this paper, two parameters, related to discrimination and noise, have been calculated. Discrimination is obtained by

$$
100\left(C_{a}-C_{s}\right) / C_{a},
$$

where $C_{a}$ and $C_{s}$ stand for the intensity of the detection peak and the intensity of the secondary highest peak, respectively. The peak-to-correlation energy ratio is defined as the intensity of the detection peak over the integrated intensity in a neighborhood. These parameters are presented in Table 1, for the four experimental JTC's considered: POF, IF, variable threshold function, and spatial envelope removal.

\section{Self-Correlation Term Removal}

One important limitation in bipolar JTC's is the presence of redundant self-correlation terms that is due to the $v$-order harmonics present in Eq. (18). In our proposed spatial envelope removal method these secondary-order terms do not appear, and consequently nondesirable redundant peaks are not present.
Optical results that compare correlations by the binary variable threshold method and by the proposed spatial envelope method are presented. The scene and the reference used are now constituted by a single satellite. Figure 10(a) displays the experimental correlation obtained with the binary JPS by the variable threshold method, showing the presence of nondesirable peaks. In Fig. 10(b) it can be seen that when the spatial envelope removal method is used the nondesirable peaks are considerably reduced.

\section{Summary}

Different kinds of nonlinear filtering in a JTC are studied and compared. The study has been realized by the use of an experimental real-time setup that operates with a single LCTV. Results obtained when POF's and IF's are used as references in the object plane are compared with the results obtained when the JPS spectrum has been binarized or processed with a simple new method for removing the spatial envelope. To avoid negative values in the incorporation of the POF or IF in object space, a holographic method of codification has been used. A normalization factor has also been chosen to optimize the optical efficiency of the process.

From the results it is clear that the experimental use of nonlinearities in Fourier space is more efficient than the use in object space by means of a POF or an IF. This is due to limitations in resolution and contrast of the LCTV available nowadays. In the future, with a better SLM it should be possible to obtain comparable results, as has been shown by the simulations. Moreover, it should be interesting to

\section{6}

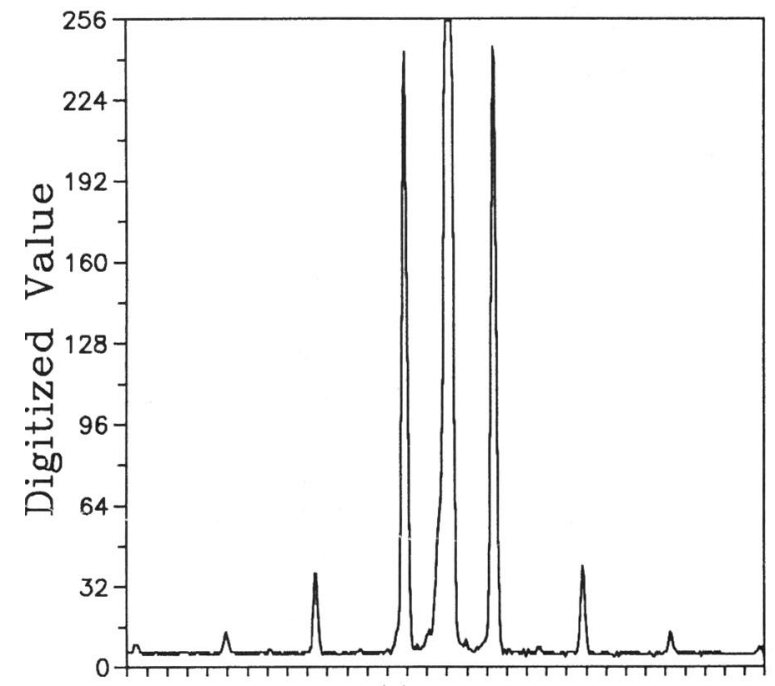

(a)

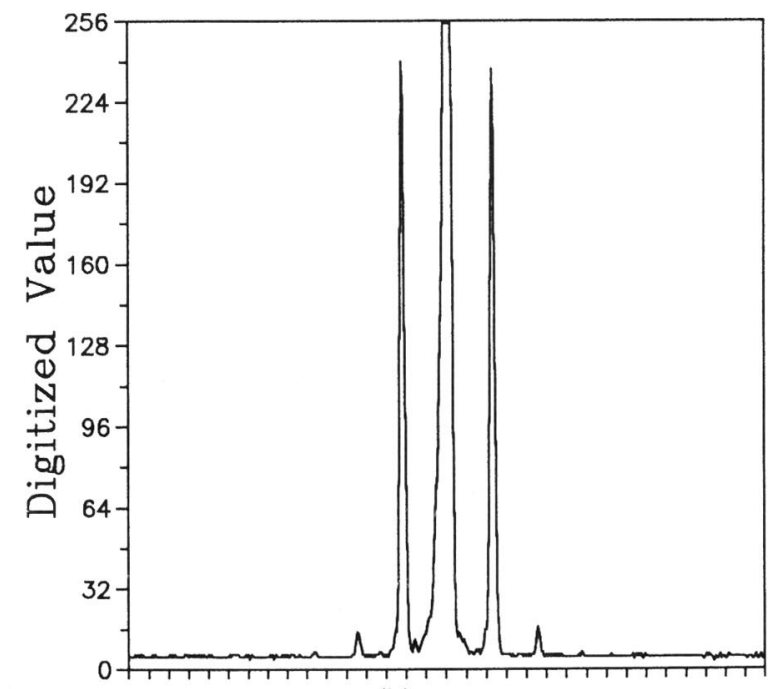

(b)

Fig. 10. Experimental correlation when the scene and the reference are a single satellite: (a) obtained with the binary JPS by the threshold function, (b) obtained by the spatial envelope removal method. Lateral peaks are eliminated. 
use other kinds of filters adapted to other situations such as synthetic discriminant functions in the object space of a JTC.

With reference to the other two types of nonlinearities analyzed in the Fourier space, the discrimination capability obtained with our spatial envelope removal method is equivalent to that obtained with the binarization of the JPS. Moreover, when the spatial envelope is removed, nondesirable redundant peaks are not present. Further advantages that should be highlighted include the simple experimental implementation and the low number of digital operations involved, which means that, in fact, optical results can be obtained in real time.

This paper has been supported in part by the Comisión Interministerial de Ciencia y Tecnología under contracts ROB91-0554 and TAP94-0303. E. Martín-Badosa gratefully acknowledges a grant from the Generalitat de Catalunya.

\section{References}

1. A. VanderLugt, "Signal detection by complex spatial filtering," IEEE Trans. Inf. Theory IT-10, 139-145 (1964).

2. H. J. Horner and P. D. Gianino, "Phase-only matched filtering," Appl. Opt. 23, 812-816 (1984).

3. G. G. Mu, X. Wang, and Z. Wang, "Amplitude compensated matched filtering," Appl. Opt. 27, 3461-3463 (1988).

4. B. Javidi, "Nonlinear joint power spectrum based optical correlation," Appl. Opt. 28, 2358-2367 (1989).
5. B. Javidi and J. Wang, "Binary nonlinear joint transfom correlation with median and subset median thresholding," Appl. Opt. 30, 967-976 (1991).

6. F. T. S. Yu, F. Cheng, T. Nagata, and D. A. Gregory, "Effects of fringe binarization of multiobject joint transform correlation," Appl. Opt. 28, 2988-2990 (1989).

7. A. Carnicer, I. Juvells, and S. Vallmitjana, "Effects of thresholding level variation in fringe binarization of multiobject joint transform correlation," Appl. Opt. 31, 1012-1024 (1992).

8. J. Wang and B. Javidi, "Multiobject detection using the binary joint transform correlator with different types of thresholding methods," Opt. Eng. 33, 1793-1805 (1994).

9. A. Carnicer, I. Juvells, and S. Vallmitjana, "Design of inverse filter for pattern recognition: analysis of the performance of an amplitude-compensated matched filter," J. Mod. Opt. 40, 391-400 (1993).

10. S. Vallmitjana, J. Campos, I. Juvells and S. Bosch, "Optical implementation of a composite filter using a joint transform correlator," in Optical Pattern Recognition II, H. J. Caulfield, ed., Proc. Soc. Photo-Opt. Instrum. Eng. 1134, 119-125 (1989).

11. W. H. Lee, "Sampled Fourier transform holograms generated by computer," Appl. Opt. 9, 639-643 (1970).

12. C. B. Burckhardt, "A simplification of Lee's method of generating holograms by computer," Appl. Opt. 9, 1949 (1970).

13. B. Javidi and J. L. Horner, "Single spatial light modulator joint transform correlator," Appl. Opt. 28, 1027-1032 (1989).

14. J. C. Kirsch, D. A. Gregory, M. W. Thie, and B. K. Jones, "Modulation characteristics of the Epson liquid crystal television," Opt. Eng. 31, 963-969 (1992).

15. T. Grycewicz, "Applying time modulation to the joint transform correlator," Opt. Eng. 33, 1813-1820 (1994).

16. A. Carnicer, E. Martín-Badosa, I. Juvells, and S. Vallmitjana, "Spatial envelop-free nonlinear joint transform correlator," Opt. Commun. 114, 336-343 (1995). 